\title{
Review of Research on Technology-Supported Cross-Cultural Learning
}

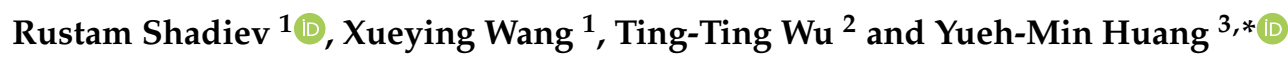 \\ 1 School of Education Science, Nanjing Normal University, Nanjing 210097, China; rustamsh@gmail.com (R.S.); \\ 180602147@njnu.edu.cn (X.W.) \\ 2 Graduate School of Technological and Vocational Education, National Yunlin University of Science and \\ Technology, Yunlin 64002, Taiwan; ttwu@yuntech.edu.tw \\ 3 Department of Engineering Science, National Cheng Kung University, Tainan 70101, Taiwan \\ * Correspondence: huang@mail.ncku.edu.tw
}

check for updates

Citation: Shadiev, R.; Wang, X.; Wu, T.-T.; Huang, Y.-M. Review of Research on Technology-Supported Cross-Cultural Learning. Sustainability 2021, 13, 1402. https:// doi.org/10.3390/su13031402

Received: 25 December 2020

Accepted: 24 January 2021

Published: 29 January 2021

Publisher's Note: MDPI stays neutral with regard to jurisdictional claims in published maps and institutional affiliations.

Copyright: (c) 2021 by the authors. Licensee MDPI, Basel, Switzerland. This article is an open access article distributed under the terms and conditions of the Creative Commons Attribution (CC BY) license (https:// creativecommons.org/licenses/by/ $4.0 /)$.

\begin{abstract}
Not many review studies have explored the theoretical foundation of cross-cultural learning or the curricula in the research they were reviewing. Furthermore, some review studies only superficially discussed the methodology and findings of the reviewed articles. To address these issues, we reviewed twenty-three studies on technology-supported cross-cultural learning published between 2014 and 2020. We aimed to summarize and analyze previous research in the following areas: (1) theoretical foundation, (2) curricula, (3) technologies, and (4) methodology and findings. Our results showed that the reviewed studies built their research framework based on diverse theoretical foundations; however, the most frequently used models were Byram's model and the cultural convergence theory. Curricula had the following main focuses: (a) cross-cultural learning, (b) linguistic skills, and (c) pre-service teacher training. The most frequently used technologies were Skype, e-mail, and blogs. We found that most reviewed studies involved the collection of both qualitative and quantitative data. Finally, most of the reviewed studies reported on the role of technologies in facilitating cross-cultural learning, FL/SL learning, and pre-service teacher training. Based on our findings, several implications along with suggestions were prepared. Our findings demonstrated that results from most studies were positive regarding technological support of crosscultural learning. Therefore, it is suggested that educators and researchers take these results into consideration when designing future studies on cross-cultural learning. Because many scholars did not report some important information, such as what theoretical foundation they built studies on or participants' demographics, we suggest that such information needs to be included in their research articles as it can be helpful in informing future studies. We also suggest that participants in future studies use variety of technological tools for supporting communication and content creation during cross-cultural learning.
\end{abstract}

Keywords: cross-cultural learning; technology; review

\section{Introduction}

With increasing contact among people from different parts of the world and the existence of cultural differences, both cultural conflicts and cultural integration occur. Therefore, it is important to be cross-culturally competent (i.e., to have the ability to understand and communicate effectively) in social encounters with people from different cultural backgrounds. Cross-cultural competence can be developed through education or academic interaction. Therefore, cross-cultural education attracted the attention of many researchers in the past.

When educators and researchers deal with the culture-related concepts, it is important to define them first. As complex concepts, culture-related terms have been defined differently by many scholars. For this present review study, we refer to culture as a set of knowledge, behaviors, attitudes, thoughts, languages, traditions, etc. that are shared 
and possessed by a group of people, and formed over many generations, that can greatly influence the behavior of group members, and which are passed down from generation to generation or acquired through learning [1,2]. Cross-cultural communication can be regarded as a process by which individuals with different cultural backgrounds communicate and exchange culture-related information with each other. Cross-cultural learning is regarded as a learning process that takes place with the help of cross-cultural communication and in which students with different cultural backgrounds learn each other's culture with or without comparing and contrasting [3]. The didactic methods involved in this process include independent learning and cooperative learning. According to Biasutti [4], cooperation refers to engagement with peers in social interaction and collaboration activities, which can help students construct their own understanding and become active learners. Furthermore, it is also important to understand different types of cross-cultural learning so that people can easily differentiate between them. The first type occurs between two or more cultures from different countries, which is the main focus of this study, e.g., learners from China learn about Irish culture, and learners from Ireland learn about Chinese culture. The second one involves two or more cultures but within one country such as religious Jews, secular Jews, and Muslims in Israel [5]. The third type involves learners from different cultures who end up residing in a country other than their own, e.g., the expat, immigrant, foreign spouse, etc.

As a part of cross-cultural education, it is suggested that people should visit some countries abroad, get immersed in the local culture for some time, and communicate with locals in order to cultivate cross-cultural abilities [6]. However, for various reasons, such as the time and money required to travel or travel restrictions and social distancing because of recent coronavirus pandemic, not many people are able to afford the above-mentioned methods for cross-cultural education. With the advancement of technology, the way we learn and communicate has gradually changed. Thus, many researchers have begun to explore the use of computer technology to support cross-cultural education without learners to physically visit the target country and communicate with representative of the target culture face to face. The technology discussed herein is mainly used to support learning and communication, e.g., to create a cross-cultural learning environment which can be regarded as a computer-supported collaborative learning environment [7], to provide authentic learning material on demand or to assist exchanging culture-related information among learners and obtaining knowledge associated with culture from individuals with different cultural backgrounds. Some examples of technology for supporting learning are learning and teaching platforms such as Blackboard, online video lectures on YouTube or open source learning resources on Wiki. One of the most commonly used techniques in computer-supported collaborative learning activities is online asynchronous discussions [7]. For instance, web 2.0 tools such as wikis allow learners to collaboratively edit and modify text, while forums are available on many platforms such as Moodle to develop online discussions [8]. Examples of technology for communication purpose are social media such as Facebook or communication tools that provide video chat and voice calls such as Skype. The use of computer technology makes it possible for people from diverse cultures to easily communicate with each other [9]. For example, it is possible to use social networks for asynchronous communication or instant messaging services for synchronous communication with representatives of a foreign country for the sake of cross-cultural learning. This interaction with members from another country brings people into direct contact with first-hand knowledge and experience [10]. As a result, technology has become very important for cross-cultural learning.

Cross-cultural learning based on technological support is especially important because of the latest pandemic during which most people have turned to online mediums for human communication. Therefore, our review study focuses on technology-supported cross-cultural learning and educators and researchers may find it intriguing to inquire more into cross-cultural learning through technological mediums and the patterns emerging from such learning. 
In this work, we review related studies on technology-supported cross-cultural learning with the aim of summarizing and analyzing studies published between 2014 and 2020 in the following four categories: (1) theoretical foundation, (2) curricula, (3) technologies, and (4) methodology and findings. This review study is important for several reasons. One reason is that while there are many review studies of this nature (see Table 1), some scholars reviewed articles published before 2014 [9,11,12]. For example, Çiftçi [9] summarized the research on technology-supported cross-cultural learning published between 2004 and 2014 with the main focus on computer-based digital technology and its effectiveness in cross-cultural learning. The main points, including satisfaction with digital tools and cross-cultural learning, increased cross-cultural knowledge, exchange between cultures, intercultural communicative competence development, issues in data analysis, etc., were reviewed in this work.

Table 1. Previous review studies: number of selected journals and articles, search terms, categories and timeframe. * technologies used to support other aspects of cross-cultural learning.

\begin{tabular}{|c|c|c|c|c|c|c|}
\hline Reference & $\begin{array}{l}\text { Number of } \\
\text { Journals }\end{array}$ & $\begin{array}{l}\text { Number of } \\
\text { Articles }\end{array}$ & $\begin{array}{l}\text { Search Terms } \\
\text { (Similar) }\end{array}$ & $\begin{array}{l}\text { Search Terms } \\
\text { (Different) }\end{array}$ & Categories & Timeframe \\
\hline Avgousti [13] & 32 & 57 & intercult *. & $\begin{array}{c}\text { telecollaborat }{ }^{*}, \\
\text { computer mediat }\end{array}$ & $\begin{array}{l}\text { 1. learner characteristics } \\
\text { 2. Web } 2.0 \text { technologies } \\
\text { 3. methodology } \\
\text { 4. challenges } \\
\text { encountered } \\
\text { 1. theories }\end{array}$ & $\begin{array}{c}\text { 2004-2015 } \\
\text { (March) }\end{array}$ \\
\hline Chun [11] & NA & NA & NA & NA & $\begin{array}{l}\text { 2. methods } \\
\text { 3. invisible factors }\end{array}$ & 1993-2014 \\
\hline Çiftçi [9] & 10 & 26 & $\begin{array}{l}\text { intercultural } \\
\text { learning, } \\
\text { cross-cultural } \\
\text { learning, } \\
\text { intercultural } \\
\text { competence, } \\
\text { intercultural } \\
\text { understanding }\end{array}$ & $\begin{array}{l}\text { intercultural } \\
\text { exchange }\end{array}$ & $\begin{array}{l}\text { 1. subjects/participants } \\
\text { 2. study duration } \\
\text { 3. technology used } \\
\text { 4. countries involved } \\
\text { 5. major findings }\end{array}$ & 2004-2014 \\
\hline $\begin{array}{c}\text { Çiftçi \& Savaş } \\
{[14]}\end{array}$ & 6 & 17 & NA & telecollaboration & $\begin{array}{l}\text { 1. focus of the study } \\
\text { 2. participants } \\
\text { 3. technologies used } \\
\text { 4. country context } \\
\text { 5. major findings }\end{array}$ & $\begin{array}{c}\text { 2010-2015 } \\
\text { (March) }\end{array}$ \\
\hline $\begin{array}{c}\text { Golonka et al. } \\
{[12]}\end{array}$ & 21 & 51 & NA & NA & $\begin{array}{l}\text { 1. technologies } \\
\text { 2. study type } \\
\text { 3. number of } \\
\text { participants } \\
\text { 4. outcome measures }\end{array}$ & 1993-2009 \\
\hline $\begin{array}{l}\text { Lewis \& O'Dowd } \\
{[15]}\end{array}$ & 24 & 54 & NA & $\begin{array}{c}\text { telecollaboration, } \\
\text { online intercultural, } \\
\text { exchange, } \\
\text { e-tandem, } \\
\text { virtual exchange }\end{array}$ & $\begin{array}{l}\text { 1. technologies } \\
\text { 2. learning aims and } \\
\text { outcomes } \\
\text { 3. countries } \\
\text { 4. number of students } \\
\text { 5. methodology }\end{array}$ & 1990-2015 \\
\hline O'Dowd [16] & 1 & 16 & NA & NA & $\begin{array}{l}\text { 1. models } \\
\text { 2. new trends }\end{array}$ & 2016 \\
\hline Piri \& Riahi [17] & 4 & 25 & $\begin{array}{l}\text { cross-culture/al, } \\
\text { interculture/al }\end{array}$ & NA & $\begin{array}{l}\text { 1. subjects/participants } \\
\text { 2. study duration } \\
\text { 3. technology used } \\
\text { 4. counties involved } \\
\text { 5. method } \\
\text { 6. maior findings }\end{array}$ & $\begin{array}{l}2007-2017 \\
\text { (October) }\end{array}$ \\
\hline
\end{tabular}

Note. The asterisk "*” is used in place of zero, single, or multiple characters. In Avgousti's [15] study, the search term telecolaborat* was used to find references containing the words telecollaboration or telecollaborative; the word intercult* yielded results such as intercultural communication, intercultural competence, intercultural communicative competence or (online) intercultural exchange; the word computer mediat* yielded results such as computer mediation, computer mediated learning or computer mediated collaborative learning.

Another reason is because some of the previous review studies (see Table 1) did not focus on technologies [11,13]. For example, Chun [11] explored the ways in which online intercultural exchange is designed, developed, and used to facilitate the develop- 
ment of both linguistic and intercultural communicative competence in higher education courses. She carried out a meta-analysis of culture-based projects and proposed four constraints: technology, configuration, learners, and learning context, in addition to the teacher's role in telecollaboration research. However, her review study lacked systematic coverage of technology-supported cross-cultural studies [14]. Avgousti [15] and Lewis and O'Dowd [16] explored technology use in the articles they reviewed. However, they only focused on technologies that support communication. That is, technologies used to support other aspects of cross-cultural learning (e.g., the creation of online cyber classrooms, learning management systems, or social networks) were neglected. Lastly, most studies did not focus on theoretical foundation and curricula [13-17].

The current study goes beyond existing review studies because we identify several issues in related studies (see above) and address them in the present study. We review papers published from 2014 up to the present to provide up-to-date information on technologysupported cross-cultural learning. We focus on different aspects of this type of learning, such as the theoretical foundation, curricula, technologies, methodology, and findings.

Another contribution of this review study is our analysis of changes in technology usage over time. Because the main focus of this review study is technology and because it is developing very quickly, it is reasonable to believe that this field might have changed in the past five years. Therefore, we analyze herein what technologies are newer and older in comparison to earlier review studies to document changes in the field over time. That is, the studies reviewed here used new technologies that were not mentioned in earlier review studies. Additionally, we list those technologies mentioned in earlier review studies that were never used for the purpose of supporting cross-cultural learning after 2014.

Therefore, the results of our review study may be helpful for educators and researchers to design and implement technology-supported cross-cultural learning projects. For example, knowing, understanding, and using an appropriate theoretical foundation are important considerations for the empirical research of educators and researchers, as well as for their integration of the theory, hypothesis, and models into pedagogical solutions related to cross-cultural learning projects. On the other hand, curriculum can provide a guiding framework for educators and researchers by which they can design and prepare instructional materials.

In this review study on technology-supported cross-cultural learning, we aimed to address the following research questions: (1) What theoretical foundation was used in the cross-cultural studies under consideration? (2) What curricula did the cross-cultural studies use? (3) What technologies were applied in the cross-cultural studies? (4) What methodology was used in the cross-cultural studies, and what were their main findings?

\section{Methods}

To ensure our search reliability, we carried out electronic and manual searches of articles. For the electronic search, we used Preferred Reporting Items for Systematic Reviews and Meta-Analyses (PRISMA). Scholars argued that PRISMA is a set of items that facilitates researchers to prepare and report various kinds of systematic reviews and meta-analyses [18]. Therefore, many scholars [19] successfully employed PRISMA in their educational studies. We used the Peer-Reviewed Instructional Materials Online Database (PRIMO) to search articles. According to Kukulska-Hulme and Viberg [20], PRIMO is a search tool and it contains several databases such as the Web of Science, ERIC, and Scopus. For this reason, PRIMO features very comprehensive collection of full-text articles and bibliographic records [21,22]. As a result, the PRIMO database has been used by many researchers in their systematic reviews and meta-analyses to find relevant literature on technology-enhanced learning [20-23].

The literature search process is shown in Figure 1. Based on general recommendations from previous review studies $[9,15,17]$, the following search terms were used in different combinations when searching for articles: technology, cross-cultural, intercultural, cultural, culture, teaching, learning, and understanding. We compiled a list of articles that match the 
search terms $(n=24,379)$. Then, we narrowed down the selection of the research articles. To this end, a list of articles was screened, and those that were not published between 2014 and 2020 were excluded from the pool $(n=14,492)$. Then, all duplicate articles were removed $(n=2754)$. Next, the following inclusion criteria were used to select articles: (1) published in English, (2) published as full texts, (3) published in the top nineteen journals (see Appendix A) related to educational technology as indexed in the Social Science Citation Index (SSCI), (4) not a review study, and (5) focused on technology-supported cross-cultural learning. The SSCI is an important channel with high authority for journal retrieval and paper references in the field of social sciences. A total of 7117 articles were excluded in the selection process. After assessing for eligibility, sixteen articles were selected. Then, the same search was conducted with the same criteria in the top nineteen journals related to educational technology indexed by the SSCI; as a result, seven additional articles were added. In total, twenty-three research articles were selected after the screening process. Two researchers were involved in this process. They independently examined all articles against the above-mentioned criteria. Differences in the screening process were identified and discussed, and eventually a consensus was reached among the researchers.

The Peer-Reviewed Instructional Materials Online Database using search terms

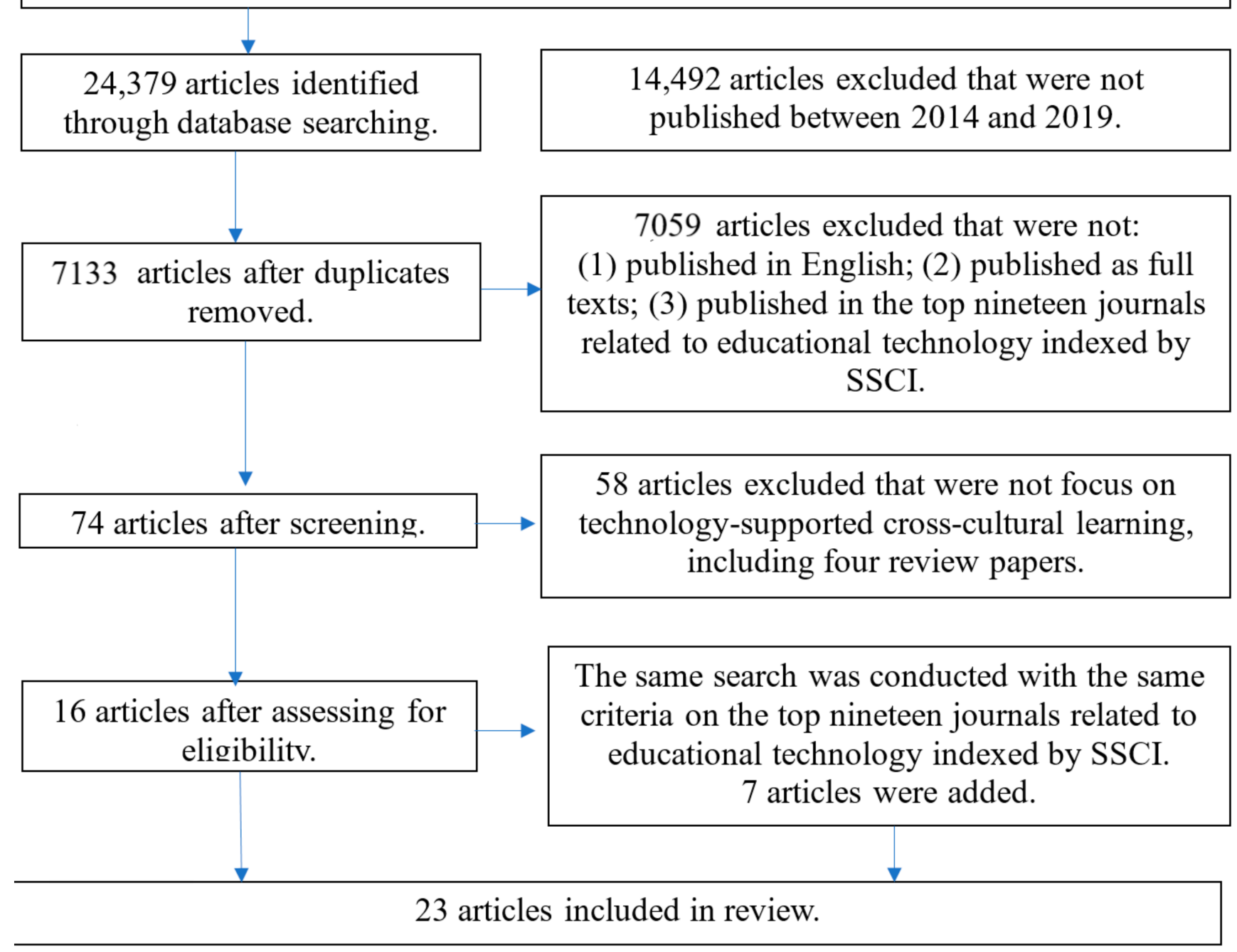

Figure 1. The literature search process.

In addition to the electronic search, we manually searched for relevant publications in the targeted journals. The manual search confirmed the articles already identified by the electronic search. 
An analytical framework (as shown in Figure 2) was proposed to answer the re-search questions and to understand the research design of the reviewed studies as well as their implementation and outcomes. The framework provided the basis for reviewing target articles and encoding research content relevant to instructional design, research design, and findings.

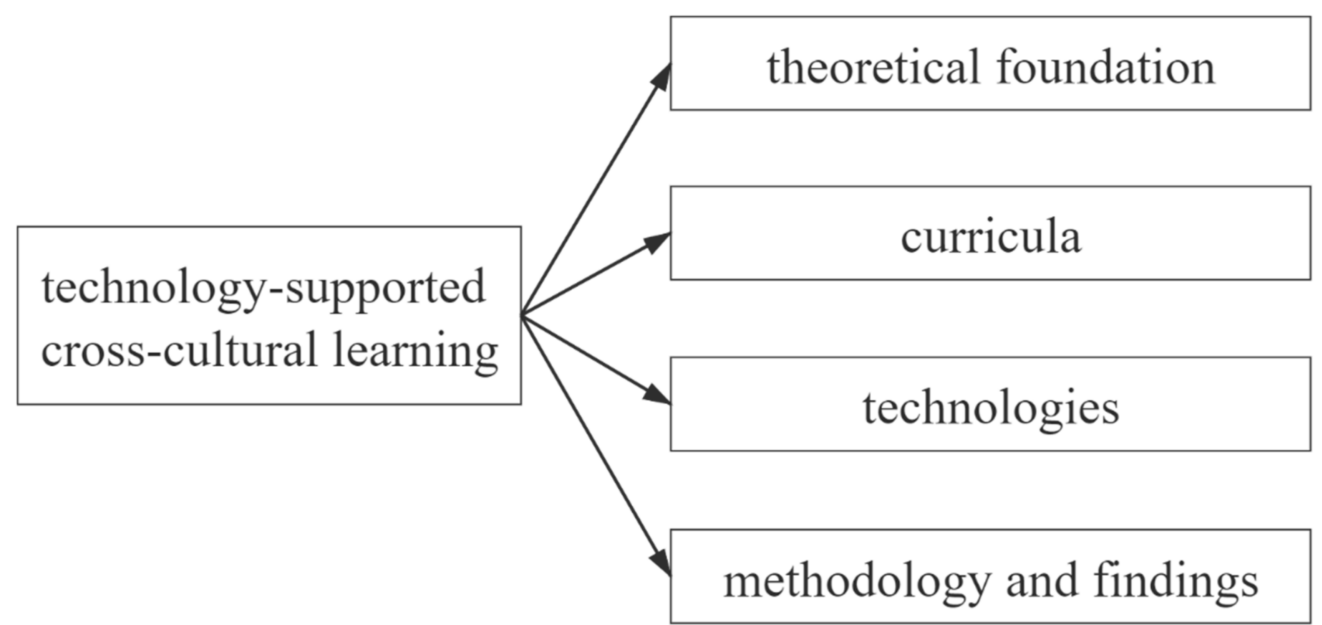

Figure 2. Analytical framework.

We carried out a content analysis using open coding [24] because our purpose was not to test hypotheses but to improve our understanding of the key issues identified in the research questions [15]. Such an approach enabled us to segment information and to form categories of information relevant to the phenomenon of interest.

Based on the general recommendations of previous studies $[9,12,14,15]$, the following coding schemes were formed: (a) theoretical foundation: the theories, models and hypothesis used as a foundation for the research, (b) curricula: the subject/s comprising a course of study for participants, (c) technologies: the tools and devices the participants used for cross-cultural learning, and (d) methodology and findings: a system of methods used in research and a conclusion reached as a result of the research. The same two researchers carried out the coding process independently. Both coders read the content of the articles independently, encoded the data by tagging concepts of interest according to the coding schemes described above, and then categorized them into groups and identified attributes for each group. After that, the coders worked together to complete the coding process and their resolved any differences or disagreements by re-examining the articles. The results of this review study are reported and discussed in the next two sections with respect to our four research questions in the following order: (1) theoretical foundation, (2) curricula, (3) technologies, and (4) methodology and findings.

\section{Results}

\subsection{Theoretical Foundation}

In this subsection, we addressed the first research question "What theoretical foundation was used in the cross-cultural studies under consideration?" Table 2 and Figure 3 present the theoretical foundation of the reviewed studies on technology-supported crosscultural learning. According to the results, no theoretical foundation was indicated in three of the reviewed studies. A total of 27 theoretical foundations were mentioned in the reviewed studies. The most frequently used theoretical foundations were Byram's model $(n=4)$ [25-28] and the cultural convergence theory $(n=4)[1,3,29,30]$. 
Table 2. Theoretical foundation.

\begin{tabular}{|c|c|c|}
\hline Theory & Frequency & Reference \\
\hline \multicolumn{3}{|l|}{ Cultural orientation } \\
\hline - Byram's model & 4 & Chen \& Yang [25], Jin [26], Chen \\
\hline 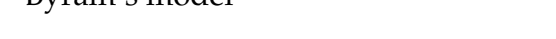 & & \& Yang [27], Hsu \& Beasley [28] \\
\hline & & Shadiev, Hwang, \& Huang [29], \\
\hline - cultural convergence theory & 4 & Shadiev \& Huang [1], Shadiev, \\
\hline & & $\begin{array}{l}\text { Wu, Sun, \& Huang [3], Shadiev, } \\
\text { Sun, \& Huang [30] }\end{array}$ \\
\hline - Byram's IC assessment guidelines & 2 & Jin [26], Hsu \& Beasley [28] \\
\hline - Allport's contact hypothesis & 1 & Chen \& Yang [27] \\
\hline - intercultural exchange model & 1 & Lee \& Markey [10] \\
\hline - Kolb's theory of experiential learning & 1 & Shadiev et al. [3] \\
\hline - reflective practice theory & 1 & Shadiev et al. [3] \\
\hline - stereotype change model & 1 & Chen \& Yang [27] \\
\hline \multicolumn{3}{|l|}{ Domain knowledge } \\
\hline - Bloom' taxonomy & 2 & Shadiev et al. [29], Yang [31] \\
\hline - constant comparative methods & 1 & Lee [32] \\
\hline - critical pedagogy & 1 & Lee [32] \\
\hline - expectancy-value theory & 1 & Chen \& Yang [27] \\
\hline - Keller's ARCS model & 1 & Chen \& Yang [27] \\
\hline - multi-literacies pedagogy & 1 & Lee [32] \\
\hline - positioning theory & 1 & $\mathrm{Wu}[33]$ \\
\hline - question classification schemes & 1 & Yang [31] \\
\hline - reflective thinking & 1 & Wach [34] \\
\hline - syntactic complexity & 1 & Schenker [35] \\
\hline - the interaction hypothesis & 1 & Schenker [35] \\
\hline - the pushed output theory & 1 & Schenker [35] \\
\hline \multicolumn{3}{|l|}{ Social lounge } \\
\hline - collaborative learning & 2 & Wach [34], Yang et al. [36] \\
\hline - social-constructivist theory & 2 & Wach [34], Yang [31] \\
\hline - dynamic systems theory & 1 & $\mathrm{Wu}[33]$ \\
\hline - interactive model & 1 & Yang \& Liao [37] \\
\hline - Community of Inquiry & 1 & Yang et al. [36] \\
\hline - PBL & 1 & Shadiev et al. [29] \\
\hline - Salmon's 5-step model & 1 & Shadiev et al. [29] \\
\hline
\end{tabular}

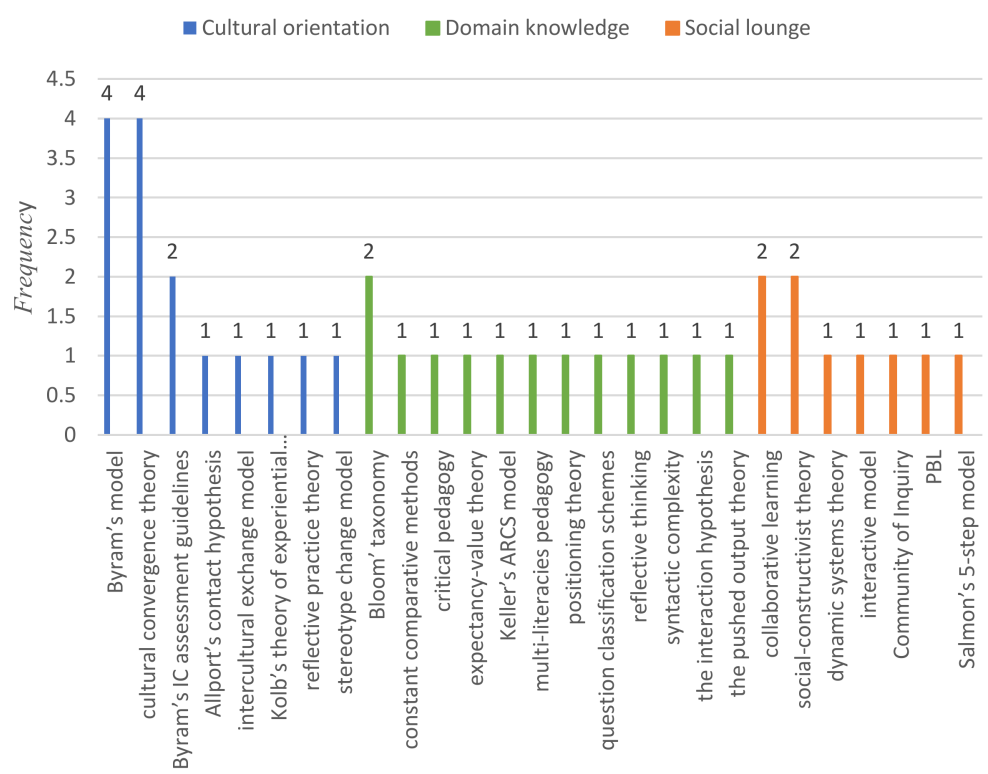

Figure 3. Frequency distribution of theory in the studies. 


\subsection{Curricula}

This subsection deal with the second research question "What curricula did the crosscultural studies use?" The data in Table 3 and Figure 4 show that the reviewed studies on technology-supported cross-cultural learning can be categorized into those that had: (1) one main focus, (2) one main focus with sub focus/es, and (3) more than one main focus. According to the results, four studies focused on cross-cultural learning only [28]. The results also showed that there were three studies with one main focus (e.g., linguistic skills) and one sub focus (e.g., cross-cultural learning) [35]. Finally, there were sixteen studies with more than one main focus, e.g., cross-cultural awareness, linguistic skills, and pre-service teacher training, in Angelova and Zhao [38].

Table 3. Curricula focus.

\begin{tabular}{|c|c|c|}
\hline Focus/es & Curricula Focus & Reference \\
\hline \multirow[t]{4}{*}{ One main focus } & -Cross-cultural competence; & Hsu \& Beasley [28] \\
\hline & -Cross-cultural understanding; & Shadiev \& Huang [1] \\
\hline & -Cross-cultural understanding; & Shadiev et al. [30] \\
\hline & -Cross-cultural competence; & Shih [2] \\
\hline \multirow{7}{*}{$\begin{array}{l}\text { One main focus with } \\
\text { sub focus/es }\end{array}$} & -Linguistic skills (Syntactic complexity); & Schenker [35] \\
\hline & $\begin{array}{l}\text {-Cross-cultural understanding; } \\
\text {-Pre-service teacher training }\end{array}$ & \\
\hline & (Teaching reflection); & Wach [34] \\
\hline & -Cross-cultural understanding; & \\
\hline & -Pre-service teacher training & \\
\hline & (Educational technology); & Yang et al. [36] \\
\hline & -Cross-cultural understanding; & \\
\hline \multirow{34}{*}{$\begin{array}{l}\text { More than one main } \\
\text { focus }\end{array}$} & -Cross-cultural awareness; & \\
\hline & -Linguistic skills; & Angelova \& Zhao [38] \\
\hline & $\begin{array}{l}\text {-Pre-service teacher training (Instruction skills); } \\
\text {-Cross-cultural competence; }\end{array}$ & \\
\hline & -Linguistic skills; & Bueno-Alastuey \& Kleban [39] \\
\hline & $\begin{array}{l}\text {-Pre-service teacher training (Instruction skills); } \\
\text {-Cross-cultural communication; }\end{array}$ & \\
\hline & -Cross-cultural competence; & Chen \& Yang [25] \\
\hline & -Linguistic skills; & \\
\hline & $\begin{array}{l}\text {-Cross-cultural competence; } \\
\text {-Linguistic skills (Language use); }\end{array}$ & Chen \& Yang [27] \\
\hline & -Cross-cultural sensitivity; & \\
\hline & -Cross-cultural understanding; & Coffey et al. [40] \\
\hline & -Cross-cultural communication; & Jin [26] \\
\hline & -Cross-cultural competence; & $\sin [20]$ \\
\hline & -Cross-cultural communication; & \\
\hline & -Cross-cultural understanding; & Lee \& Markey [10] \\
\hline & -Linguistic skills (Linguistic awareness); & \\
\hline & -Cross-cultural competence; & Lee \& Park [41] \\
\hline & -Linguistic skills; & Lee \& Park [41] \\
\hline & -Cross-cultural communication; & \\
\hline & -Cross-cultural understanding; & Lee [32] \\
\hline & -Linguistic skills (language use); & \\
\hline & $\begin{array}{l}\text {-Cross-cultural communication; } \\
\text {-Linguistic skills; }\end{array}$ & Liao \& Lu [42] \\
\hline & -Cross-cultural communication; & \\
\hline & -Cross-cultural competence; & Melo-Pfeifer [43] \\
\hline & -Linguistic skills; & \\
\hline & -Cross-cultural communication; & Shadiev et al. [29] \\
\hline & -Cross-cultural understanding; & Shadiev et al. [29] \\
\hline & -Cross-cultural sensitivity; & Shadiev et al. [3] \\
\hline & -Cross-cultural understanding; & snadiev et al. [3] \\
\hline & -Cross-cultural understanding; & \\
\hline & -Linguistic skills (writing skills); & Wu [33] \\
\hline & -Cross-cultural communication; & \\
\hline & -Cross-cultural understanding; & Yang [31] \\
\hline & -Linguistic skills; & \\
\hline & $\begin{array}{l}\text {-Cross-cultural communication; } \\
\text {-Cross-cultural understanding. }\end{array}$ & Yang \& Liao [37] \\
\hline
\end{tabular}




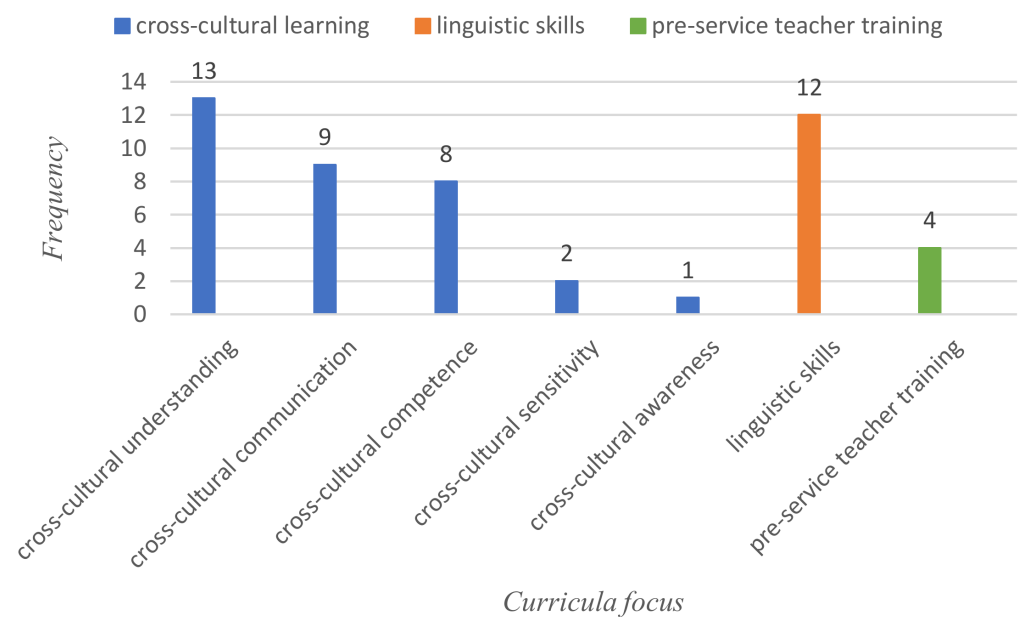

Figure 4. Frequency distribution of curricula focus in the studies.

A further data analysis revealed that the curricula focus in all of the reviewed studies could be divided into three main areas, the first of which is cross-cultural learning $(n=23)$, including several aspects: cross-cultural understanding $(n=13)$, cross-cultural communication $(n=9)$, cross-cultural competence $(n=8)$, cross-cultural sensitivity $(n=2)$, and cross-cultural awareness $(n=1)$. The second is linguistic skills $(n=12)$, such as communication skills $(n=4)$ and linguistic awareness $(n=1)$. The third area includes pre-service teacher training $(n=4)$, for example, instructional skills $(n=2)$, especially grammar instruction skills, techno-pedagogical skills, and teaching reflection $(n=1)$, and a major curriculum related to teaching, e.g., educational technology $(n=1)$.

\subsection{Technologies}

The following research question "What technologies were ap-plied in the cross-cultural studies?" was addressed in this subsection. Table 4 and Figure 5 show the types of technologies used in the reviewed articles. According to the results, there were nineteen technological tools, among which the most frequently used was Skype $(n=7)$, followed sequentially by e-mail $(n=6)$, blogs $(n=5)$, virtual environments $(n=4)$, Facebook $(n=4)$, and speech-enabled language translation $(n=3)$. Next on the list were technologies such as discussion boards $(n=2)$, videoconferencing $(n=2)$, online forums $(n=2)$, iMovie or Movie Maker $(n=2)$, Wiki $(n=2)$, and WeChat/QQ $(n=2)$, sequentially. The technologies used only once $(n=1)$ were Blackboard, Twitter, Podcasts, the Join Net ${ }^{\mathrm{TM}}$, Moodle, and Dropbox.

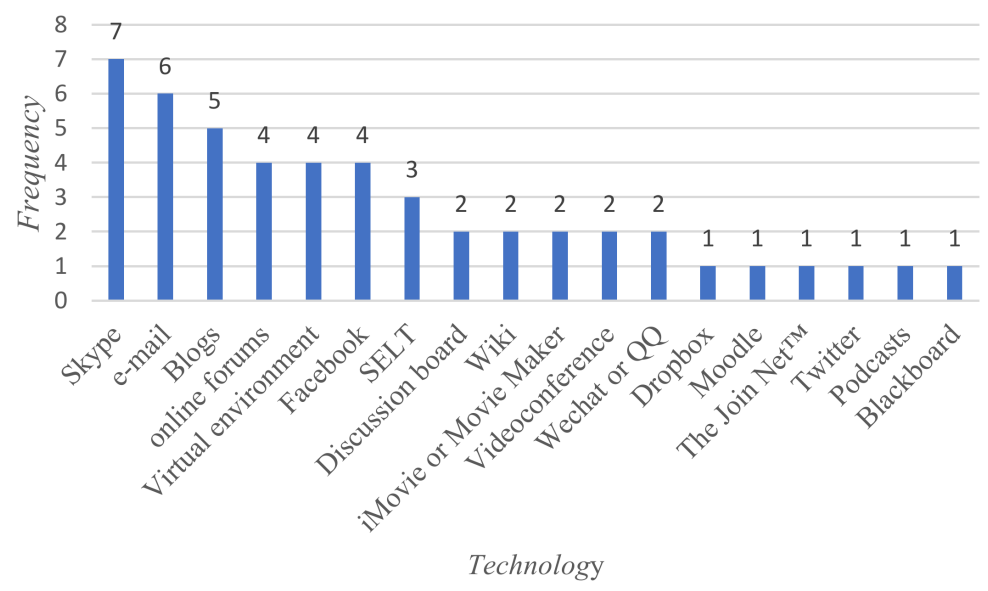

Figure 5. Frequency distribution of technology used in the studies.

The types of technology used before and after 2014 are presented in Table 5. We found that compared with the types of technological tools used in related studies before 
2014, some new technologies have been applied to support cross-cultural learning. These include, for example, Facebook, Skype, the Join Net ${ }^{\mathrm{TM}}$, speech-enabled language translation, virtual environments, and WeChat/QQ. On the other hand, some of the tools mentioned in previous review studies are no longer in use in studies published after 2014, for example, Twitter and Podcasts.

Table 4. Technology used.

\begin{tabular}{|c|c|c|}
\hline Technology & Frequency & Reference \\
\hline Skype & 7 & $\begin{array}{c}\text { Angelova \& Zhao [38], Shadiev et al. [3], } \\
\text { Shadiev et al. [30], Bueno-Alastuey \& } \\
\text { Kleban [39], Chen \& Yang [25], Hsu \& } \\
\text { Beasley [28], Lee [32] }\end{array}$ \\
\hline e-mail & 6 & $\begin{array}{c}\text { Bueno-Alastuey \& Kleban [39], Chen \& } \\
\text { Yang [25], Hsu \& Beasley [28], Lee \& } \\
\text { Park [41], Schenker [35], Wach [34] }\end{array}$ \\
\hline Blogs & 5 & $\begin{array}{l}\text { Chen \& Yang [25], Lee \& Markey [10], } \\
\text { Melo-Pfeifer [43], Schenker [35], Shih [2] }\end{array}$ \\
\hline online forums & 4 & $\begin{array}{c}\text { Shadiev \& Huang [1], Chen \& Yang [25], } \\
\text { Wu [33], Yang [31] }\end{array}$ \\
\hline Virtual environment & 4 & $\begin{array}{c}\text { Coffey et al. [40], Liao \& Lu [42], Shih [2], } \\
\text { Yang \& Liao [37] }\end{array}$ \\
\hline Facebook & 4 & $\begin{array}{c}\text { Shadiev et al. [3], Shadiev et al. [30], } \\
\text { Jin [26], Lee [32] }\end{array}$ \\
\hline SELT & 3 & $\begin{array}{l}\text { Shadiev \& Huang [1], Shadiev et al. [3], } \\
\text { Shadiev et al. [30] }\end{array}$ \\
\hline Discussion board & 2 & Angelova \& Zhao [38], Shadiev et al. [29] \\
\hline Wiki & 2 & Chen \& Yang [27], Lee [32] \\
\hline iMovie or Movie Maker & 2 & Lee \& Markey [10], Lee [32] \\
\hline Videoconference & 2 & Lee \& Park [41], Schenker [35] \\
\hline Wechat or QQ & 2 & Liao \& Lu [42], Yang [31] \\
\hline Dropbox & 1 & Bueno-Alastuey \& Kleban [39] \\
\hline Moodle & 1 & Chen \& Yang [27] \\
\hline The Join $\mathrm{Net}^{\mathrm{TM}}$ & 1 & Shadiev et al. [29] \\
\hline Twitter & 1 & Lee \& Markey [10] \\
\hline Podcasts & 1 & Lee \& Markey [10] \\
\hline Blackboard & 1 & Yang et al. [36] \\
\hline
\end{tabular}

Table 5. Technology used before 2014 and/or after 2014.

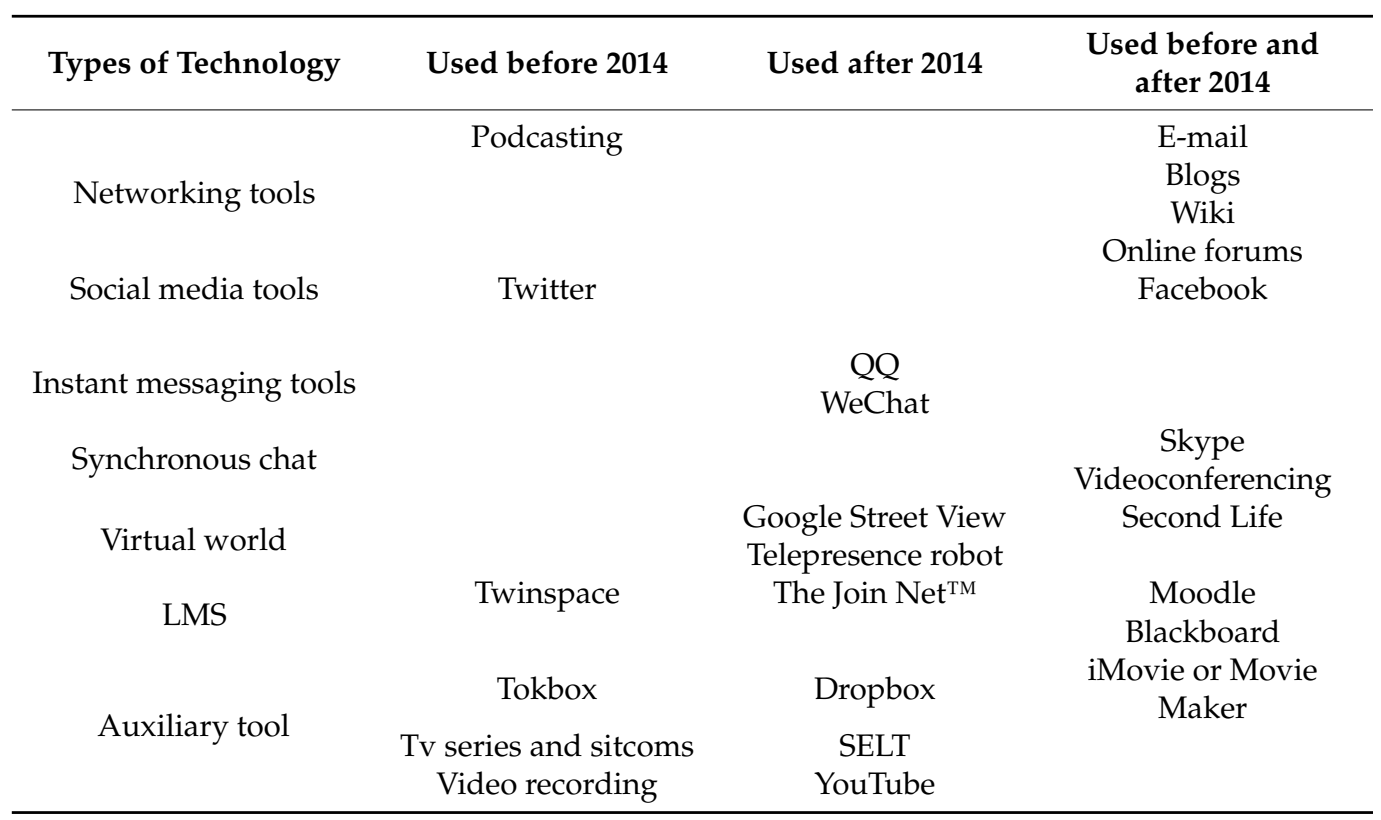




\subsection{Methodology and Findings of Studies Reviewed}

In this Section, we deal with the fourth research question of the study "What methodology was used in the cross-cultural studies, and what were their main findings?" Table 6 shows the participants' academic level and age. According to the results, seventeen studies were carried out with higher education students aged from seventeen to forty-four. Scholars in seven studies did not show the age of their higher education students. Some studies, e.g., Angelova \& Zhao [38], involved participants from two countries, but scholars provided demographic information on participants only from one country. Six studies were carried out with elementary and secondary school students ranging in age from ten to eighteen. Scholars in one study did not show the age of their participants. One study had participants from university and secondary school. Finally, there was one study in which scholars did not show participants' academic level or age.

Table 6. Participants' educational level and age.

\begin{tabular}{ccc}
\hline Academic Status & Age & Reference \\
\hline & $17-19$ & Angelova \& Zhao [38] \\
$18-22$ & Yang [31] \\
$18-23$ & Schenker [35] \\
$18-44$ & Yang et al. [36] \\
$19-22$ & Jin [26] \\
university & $19-24$ & Bueno-Alastuey \& Kleban [39] \\
& $20-32$ & Shadiev et al. [30] \\
& $20-35$ & Shadiev et al. [29] \\
& $22-26$ & Yang \& Liao [37] \\
$24-28$ & Liao \& Lu [42] \\
& NA & Beasley [28], Lee \& Markey [10], Shih [2], \\
& $10-15$ & Wach [34], Wu [33] \\
& $12-15$ & Lee \& Park [41] \\
elementary and secondary school & Chen \& Yang [27] \\
& $14-15$ & Lee [32] \\
& $16-18$ & Shadiev \& Huang [1] \\
& NA & Schenker [35] \\
& NA & Chen \& Yang [25] \\
& & Melo-Pfeifer [43] \\
\hline
\end{tabular}

Table 7 illustrates the data collection method, and Table 8 presents the main findings of the reviewed studies. According to the results, there were studies that adopted qualitative and quantitative $(n=13)$ methodologies or only qualitative $(n=9)$ methodologies, and there was one study that only used a quantitative method.

According to the results, most studies clearly pointed out how cross-cultural learning is enhanced by the use of technologies $(n=20)$. Most studies also reported on the usefulness of technology for FL/SL learning $(n=13)$ carried out in combination with cross-cultural learning activities. Several studies reported advancements in the learners' instructional skills $(n=4)$. In addition, there were results related to learner perceptions (e.g., perceptions of technology or cross-cultural projects). Most of these results were positive. In addition, several studies indicated problems or challenges encountered in the process of using technology in cross-cultural learning and offered solutions $(n=8)$. Some other discoveries $(n=9)$, including useful strategies for cross-cultural online collaborative learning or error correction, learner communication types in the process of cross-cultural learning, and some factors affecting cultural learning, were also reported. 
Table 7. Data collection.

\begin{tabular}{|c|c|}
\hline Data Collection & Reference \\
\hline $\begin{array}{l}\text { QL: postings, papers, essays, interviews } \\
\text { QT: essays }\end{array}$ & Angelova \& Zhao [38] \\
\hline $\begin{array}{l}\text { QL: open-ended questions } \\
\text { QT: close-ended questions }\end{array}$ & Bueno-Alastuey \& Kleban [39] \\
\hline QL: interviews, document analysis & \\
\hline $\begin{array}{l}\text { QT: pre-survey/mid-term survey/post survey, } \\
\text { reflective surveys }\end{array}$ & Chen \& Yang [25] \\
\hline $\begin{array}{l}\text { QL: online communication, pre-survey/post survey } \\
\text { QT: pre-survey/post survey }\end{array}$ & Chen \& Yang [27] \\
\hline $\begin{array}{l}\text { QL: interviews } \\
\text { QT: pre/post-test, delayed post-test }\end{array}$ & Coffey et al. [40] \\
\hline $\begin{array}{l}\text { QL: interviews; reflective journals } \\
\text { QT: post-project questionnaire }\end{array}$ & Hsu \& Beasley [28] \\
\hline QL: observations, interviews & Jin [26] \\
\hline $\begin{array}{l}\text { QL: comments } \\
\text { QT: pre-survey/post survey, open-ended questions }\end{array}$ & Lee \& Markey [10] \\
\hline QT: survey & Lee \& Park [41] \\
\hline QL: the author's field notes, questionnaire, class artifacts & Lee [32] \\
\hline QL: interviews, field notes, video interactions; & Liao \& Lu [42] \\
\hline QL: posts, comments & Melo-Pfeifer [43] \\
\hline $\begin{array}{l}\text { QL: e-mail and blog data } \\
\text { QT: e-mails }\end{array}$ & Schenker [35] \\
\hline QL: online communication, interviews, questionnaire & Shadiev \& Huang [1] \\
\hline QL: interviews, messages, reflections & Shadiev et al. [29] \\
\hline $\begin{array}{l}\text { QL: online communication, interviews } \\
\text { QT: pre/post-test, scale, questionnaire }\end{array}$ & Shadiev et al. [3] \\
\hline QL: online communication, interviews & Shadiev et al. [30] \\
\hline $\begin{array}{l}\text { QL: observation, interviews, reflective posts } \\
\text { QT: tests }\end{array}$ & Shih [2] \\
\hline $\begin{array}{l}\text { QL: e-mails, presentations, an open-ended question, } \\
\text { descriptive comments } \\
\text { QT: close-ended questions }\end{array}$ & Wach [34] \\
\hline QL: posts, reflective essays, interviews & $\mathrm{Wu}[33]$ \\
\hline $\begin{array}{l}\text { QL: interviews } \\
\text { QT: pre/post-test, questionnaires }\end{array}$ & Yang \& Liao [37] \\
\hline QL: pre/post-survey, questionnaire, text chat logs & Yang [31] \\
\hline $\begin{array}{l}\text { QL: open-ended questions; posts; the focus group interviews } \\
\text { QT: close-ended questions }\end{array}$ & Yang et al. [36] \\
\hline
\end{tabular}

Table 8. Main findings with respect to three dimensions (i.e., cultural orientation, domain knowledge, social lounge).

\begin{tabular}{|c|c|c|c|}
\hline Cultural Orientation & Domain Knowledge & Social Lounge & Reference \\
\hline Develop cross-cultural awareness & $\begin{array}{l}\text { Improve grammar } \\
\text { instruction skills; } \\
\text { Improve English } \\
\text { language skills. }\end{array}$ & & Angelova \& Zhao [38] \\
\hline $\begin{array}{l}\text { Intercultural competence; } \\
\text { The design and implementation } \\
\text { of the cross-cultural project: } \\
\text { positive (several shortcomings) }\end{array}$ & $\begin{array}{l}\text { Linguistic gains; } \\
\text { Techno-pedagogical } \\
\text { skills advantages. }\end{array}$ & $\begin{array}{c}\text { No student mention anything } \\
\text { negative about the choice } \\
\text { of ICT }\end{array}$ & Bueno-Alastuey \& Kleban [39] \\
\hline $\begin{array}{l}\text { Students' learning benefits from } \\
\text { technology-enhanced } \\
\text { intercultural language learning } \\
\text { (TEILI) project: foster ICC; } \\
\text { Students' attitudes towards TEILI } \\
\text { project: strongly positive; } \\
\text { Students' evaluations of TEILI } \\
\text { project: helpful. }\end{array}$ & $\begin{array}{l}\text { Foster linguistic } \\
\text { competence; } \\
\text { Students' self-perceived } \\
\text { challenges: accent, } \\
\text { vocabulary; } \\
\text { Teaching reflections } \\
\text { on TEILI. }\end{array}$ & & Chen \& Yang [25] \\
\hline
\end{tabular}


Table 8. Cont.

\begin{tabular}{|c|c|c|c|}
\hline Cultural Orientation & Domain Knowledge & Social Lounge & Reference \\
\hline Facilitate the development of ICC & $\begin{array}{l}\text { Students' perceptions of } \\
\text { language use: } \\
\text { contradictory feelings }\end{array}$ & $\begin{array}{l}\text { The students' attitudes } \\
\text { toward and evaluation of the } \\
\text { UBOD: positive }\end{array}$ & Chen \& Yang [27] \\
\hline $\begin{array}{c}\text { Improve students' } \\
\text { cross-cultural sensitivity; } \\
\text { An interaction effect for channel } \\
\text { and gender pertaining to } \\
\text { intercultural sensitivity. }\end{array}$ & & \multirow[t]{2}{*}{$\begin{array}{l}\text { A channel effect of the Second } \\
\text { Life environment }\end{array}$} & Coffey et al. [40] \\
\hline $\begin{array}{l}\text { Strong positive perceptions and } \\
\text { attitudes toward } \\
\text { intercultural CMC; } \\
\text { Develop students' IC; } \\
\text { Some important sources in } \\
\text { avoiding being overwhelmed. }\end{array}$ & & & Hsu \& Beasley [28] \\
\hline $\begin{array}{l}\text { Facilitate intercultural interactions } \\
\text { and intercultural competence }\end{array}$ & & $\begin{array}{l}\text { Facebook is useful to facilitate } \\
\text { intercultural interactions and } \\
\text { intercultural competence in } \\
\text { the EFL classroom }\end{array}$ & Jin [26] \\
\hline $\begin{array}{l}\text { Affordances and challenges of } \\
\text { intercultural exchange; } \\
\text { Effects of intercultural task type } \\
\text { and topic choice: positive. }\end{array}$ & $\begin{array}{l}\text { Role of peer feedback and } \\
\text { strategies for error } \\
\text { correction related } \\
\text { to linguistic }\end{array}$ & \multirow[t]{3}{*}{$\begin{array}{l}\text { Web } 2.0 \text { tools: satisfied, } \\
\text { Twitter's limitations }\end{array}$} & Lee \& Markey [10] \\
\hline $\begin{array}{l}\text { Self-efficacy (SE), exchange } \\
\text { infrastructure (EI) and quality of } \\
\text { exchange activities (QEA) have a } \\
\text { significant effect on the students' } \\
\text { learning satisfaction and } \\
\text { intercultural competence }\end{array}$ & $\begin{array}{l}\text { With considerable impact } \\
\text { resulting from EI and } \\
\text { QEA, the students' SE and } \\
\text { EI further affected the } \\
\text { improvement of their } \\
\text { foreign } \\
\text { language capability }\end{array}$ & & Lee \& Park [41] \\
\hline $\begin{array}{l}\text { The advantages of students' } \\
\text { learning experiences in the } \\
\text { computer-mediated } \\
\text { intercultural communication }\end{array}$ & $\begin{array}{l}\text { Pedagogical challenges } \\
\text { and a high cost of its } \\
\text { implementation; } \\
\text { Propose three teaching } \\
\text { and instructional } \\
\text { principles to } \\
\text { address question. }\end{array}$ & & Lee [32] \\
\hline $\begin{array}{l}\text { Authentic cross-cultural } \\
\text { learning experience }\end{array}$ & $\begin{array}{l}\text { Foster foreign language } \\
\text { learning outdoors; } \\
\text { Useful implications for } \\
\text { future research design. }\end{array}$ & $\begin{array}{l}\text { Perceived benefits and } \\
\text { challenges of using } \\
\text { Tele-presence robots }\end{array}$ & Liao \& Lu [42] \\
\hline $\begin{array}{l}\text { Facilitate cross-cultural } \\
\text { competence }\end{array}$ & $\begin{array}{l}\text { Develop plurilingual } \\
\text { competence and } \\
\text { linguistic skills }\end{array}$ & $\begin{array}{l}\text { Some benefits of } \\
\text { pedagogical blogs }\end{array}$ & Melo-Pfeifer [43] \\
\hline & $\begin{array}{l}\text { Syntactic complexity in L2 } \\
\text { learners' e-mails change; } \\
\text { Three main factors that } \\
\text { impact the increase of } \\
\text { syntactic complexity. }\end{array}$ & & Schenker [35] \\
\hline Cross-cultural learning took place & & $\begin{array}{l}\text { The two systems are easy to } \\
\text { use and useful for } \\
\text { cross-cultural learning; } \\
\text { The texts produced are } \\
\text { acceptable and useful. }\end{array}$ & Shadiev \& Huang [1] \\
\hline $\begin{array}{l}\text { Facilitate cross-cultural learning; } \\
\text { Students' cross-cultural } \\
\text { communication type: educational } \\
\text { (useful), technical, } \\
\text { communicative. }\end{array}$ & & $\begin{array}{l}\text { A PBCL approach in the } 3 C \\
\text { online environment: useful; } \\
\text { Students' perceptions: } \\
\text { positive. }\end{array}$ & Shadiev et al. [29] \\
\hline
\end{tabular}


Table 8. Cont

\begin{tabular}{|c|c|c|c|}
\hline Cultural Orientation & Domain Knowledge & Social Lounge & Reference \\
\hline $\begin{array}{l}\text { Facilitate the cross-cultural } \\
\text { understanding of the participants; } \\
\text { Enhance intercultural sensitivity. }\end{array}$ & & $\begin{array}{c}\text { Speech-enabled language } \\
\text { translation system evaluation: } \\
\text { meaningful, useful; } \\
\text { Students' perceptions: } \\
\text { positive } \\
\text { The accuracy rate for different } \\
\text { language is different, } \\
\text { improving, and some } \\
\text { influencing factors; } \\
\text { Some issues related to STR } \\
\text { and CAT processes } \\
\text { and solutions. }\end{array}$ & Shadiev et al. [3] \\
\hline $\begin{array}{l}\text { Increase cultural knowledge; } \\
\text { The development of attitudes } \\
\text { toward the target } \\
\text { culture: positive; } \\
\text { The possible learner factors affect } \\
\text { culture learning. }\end{array}$ & & & Shih [2] \\
\hline $\begin{array}{l}\text { High levels of overall satisfaction } \\
\text { with the cross-cultural project; } \\
\text { Benefit from taking part in the } \\
\text { project in many ways. } \\
\text { Facilitate cross-cultural } \\
\text { understanding; }\end{array}$ & $\begin{array}{l}\text { Stimulate teaching } \\
\text { reflections }\end{array}$ & & Wach [34] \\
\hline $\begin{array}{c}\text { Positioning (mis)alignment in } \\
\text { intercultural asynchronous } \\
\text { computer-mediated } \\
\text { communication (ACMC); } \\
\text { Pedagogical practices for } \\
\text { intercultural ACMC activities. } \\
\text { Improve the cultural }\end{array}$ & Develop writing skills. & $\begin{array}{c}\text { Discursive practices, } \\
\text { positioning systems and } \\
\text { its properties. }\end{array}$ & $\mathrm{Wu}[33]$ \\
\hline $\begin{array}{l}\text { learning effectiveness; } \\
\text { Promote interpersonal } \\
\text { communication between teachers } \\
\text { and students. }\end{array}$ & & $\begin{array}{l}\text { Verify the usability of the } \\
\text { VECAR: positive but with } \\
\text { some problems }\end{array}$ & Yang \& Liao [37] \\
\hline $\begin{array}{l}\text { Facilitate cross-cultural } \\
\text { understanding }\end{array}$ & $\begin{array}{l}\text { Develop language skills; } \\
\text { Self-generate many } \\
\text { higher-order thinking } \\
\text { questions; } \\
\text { The difference of asking } \\
\text { thinking questions } \\
\text { between Chinese English } \\
\text { learners and their } \\
\text { U.S. counterparts. }\end{array}$ & & Yang [31] \\
\hline $\begin{array}{l}\text { Students' collaboration process } \\
\text { was influenced by culture; } \\
\text { Students were interested in each } \\
\text { other's culture; } \\
\text { Students' attitude towards the } \\
\text { cross-cultural online collaborative } \\
\text { learning experience is positive; } \\
\text { Four strategies to conduct smooth } \\
\text { and effective cross-cultural online } \\
\text { collaborative learning. }\end{array}$ & $\begin{array}{l}\text { Students' collaboration } \\
\text { process is influenced } \\
\text { by language. }\end{array}$ & & Yang et al. [36] \\
\hline
\end{tabular}




\section{Discussion}

\subsection{Theoretical Foundation}

In this Section, according to the first research question "What theoretical foundation was used in the cross-cultural studies under consideration?" we discuss the theoretical foundations and their main dimensions, and our suggestions for educators and researchers. According to the results, the theoretical foundations used by scholars for their studies on technology-supported cross-cultural learning cover three different dimensions: cultural orientation (i.e., situations when the participants learn about each other's culture), domain knowledge (i.e., major topics related to the learning content in a specific course such as linguistic skills or pre-service training that are discussed by the participants), and social lounge (i.e., an environment in which the participants attempt to become better acquainted by introducing themselves using basic personal information and descriptions of their cultural background). Scholars aimed to base their research on various theoretical foundations for learners to learn about a foreign culture (i.e., cultural orientation), to obtain knowledge of some specific domain such as linguistics (i.e., domain knowledge), and to create online learning environments (i.e., social lounge). For example, Chinese students communicated with peers from Canada, Lebanon, the Netherlands, and Ghana in English as a foreign language and also learned about their cultures [27]. Therefore, this project facilitated their linguistic and intercultural competencies. In addition, scholars created online learning environments to support the learning process as well as communication because the learners were from different countries and could not meet face to face. For example, Jin [26] adopted Facebook to create an online learning environment in which South Korean students communicated with students from the US.

The most frequently used theoretical foundations were Byram's model and the cultural convergence theory. Byram [44] advocated that to become cross-culturally competent, learners need to acquire the following: (1) knowledge of their own and foreign cultures and general processes related to cultural practices, products, and interactions, (2) attitudes such as curiosity, openness, and readiness to learn about other cultures, (3) interpreting and relating skills, which are defined as the ability to explain and identify cultural perspectives and to mediate between them, (4) discovery and interaction skills, which are defined as skills related to the ability to acquire new knowledge about a culture and cultural practices and apply it in real-time communication and interaction, and (5) cultural awareness, which refers to the ability to critically evaluate the perspectives, practices, and products of a person's own culture and that of a foreign culture. According to the cultural convergence theory, cross-cultural learning takes place when two or more learners from different cultures reach a mutual understanding of each other's culture and the world in which they live through communication and information exchange [3]. In other words, experiences and insights into each other's cultures that learners communicate and share among themselves enable them to expand their cultural awareness and behavior [36]. Following the notion of this theory, Coffey et al. [40], Hsu and Beasley [28], and Yang [31] suggest that cross-cultural learning can be successfully achieved when a learner has the following competencies: (1) knowledge, i.e., familiarization with different cultural characteristics, values, beliefs, and behaviors, (2) awareness, i.e., an internal understanding and appreciation of a culture, (3) sensitivity, i.e., the ability to read into situations, contexts, and behaviors that are culturally rooted and to react to them appropriately, and (4) competence, i.e., the ability to work effectively across cultures, including such things as respecting cultural differences, adapting to changing situations, and benefiting from them.

Some of our findings are in line with those from earlier review studies. For example, the finding related to the most frequently used theoretical model (i.e., Byram's model) is in line with review results of Chun [11] and Çiftçi [9].

Based on our results, we have several suggestions for educators and researchers. First, we suggest that scholars indicate what theoretical foundation was used in their research. This information is essential because it helps readers understand the theoretical background based on which the research was designed. Golonka et al. [12] reported similar issues in 
their review study and argued that some studies had poor descriptions of the research design, which made evaluation of the efficacy of technology-assisted learning problematic.

Second, although Byram's model and the cultural convergence theory were frequently used in research on cross-cultural learning it should be noted where these theories have been developed (i.e., mostly in the so-called Western world), so they may not capture complexities of human communication that take place in different parts of the world with different histories and worldviews.

Third, we suggest considering other theoretical foundations as well. For example, Byram's model and the cultural convergence theory focus solely on learners and their cognitive processes and other important factors involved in the cross-cultural learning process (e.g., the role of the instructor and/or social behavior) are ignored. The community of inquiry model $[45,46]$, which considers not only cognitive presence but also teaching and social presence, might be a good model for designing online cross-cultural learning projects. In terms of teaching presence, future studies may consider designing and managing learning sequences, providing subject-matter expertise, and facilitating active learning [46]. With respect to social presence, scholars may wish to focus on the ability of learners to project themselves socially and emotionally in learning activities [45]. The contact hypothesis can be used for the second type of cross-cultural learning that was mentioned in the Introduction section (i.e., between two or more cultures within one country). According to Allport [47], intergroup contact under appropriate conditions is one of the most effective ways to reduce prejudice between majority and minority group members. For example, through intergroup contact, a learner can change stereotypes related to his peers from a different culture [27]. Walther et al. [5] focused on inter-cultural learning and successfully used the contact hypothesis in their studies; however, their studies were out of our scope, and therefore not included in this review research.

Furthermore, for future review studies, we suggest that researchers use more/different search terms and selection criteria to locate more research articles that have used the contact hypothesis. Because this hypothesis is relevant and important for cross-cultural learning, summarizing such articles would be useful for the community.

\subsection{Curricula}

Research questions for this Section was "What curricula did the cross-cultural studies use?" In studies on cross-cultural understanding supported by technology, scholars aimed their efforts at facilitating the acquisition of an understanding of different cultures on the part of learners [40]. In studies on cross-cultural communication, learners communicated and exchanged culturally-related information with each other, and scholars explored how learners from differing cultural backgrounds communicate [25]. Studies with a focus on cross-cultural competence created technology-supported environments in which learners from different cultures could understand each other's culture, view cultural differences, and develop good critical cultural awareness by communicating information related to their respective countries or cultures with an open and tolerant attitude [39], where the primary goal of cross-cultural learning was to develop learners' cross-cultural competence [26,28]. In studies on cross-cultural sensitivity, learners acquired culture-related information to improve their ability to read into situations, contexts, and behaviors and then to react to them appropriately [3]. In studies focused on cross-cultural awareness, students learned about a foreign culture to both understand and appreciate it [38].

In studies with a focus on linguistic skills supported by technology, scholars explored learners' communication skills and linguistic awareness [33]. For the purpose of improving communication skills (i.e., writing), language learners communicated with speakers of the target language with a focus on syntactic complexity [35] or grammatical structure [43]. In a study conducted by Lee [32], language learners and speakers of the target language also interacted with each other, but these scholars all focused on communication skills, including reading, writing, listening, and speaking. In this case, language learners were able to experience the use of language in real situations and reflect on their experiences. 
In order to improve linguistic awareness, language learners and speakers of the target language communicated with each other, and speakers of the target language pointed out mistakes and issues in the communicated content of the language learners so that the learners became aware of their own mistakes [10]. Scholars focused in their curricula on both linguistic and cross-cultural skills because language learning cannot be isolated from cultural learning [38]. According to the related studies, language learners should familiarize themselves with the target culture [35]. This helps "entail the interface between language and culture by implicitly referring to linguistic, sociolinguistic and pragmatic competences that lead to understanding of otherness" [38].

Several studies focused on pre-service teacher training using technologies. That is, scholars created opportunities for pre-service teachers to practically test their previously learned theoretical knowledge, so as to improve their instructional skills, engage in reflection, and practice their knowledge of curricula specifically related to teaching. Collaborative learning activities among representatives of different cultures were designed during the pre-service teacher training sessions. The aim was to adjust instruction to diverse contexts and learners [34] and improve teaching skills [39], as well as to help pre-service teachers acquire multi-cultural awareness and cross-cultural collaborative skills before they embarked on teaching in a real-world environment [36]. For example, two studies aimed at improving the participants' linguistic instruction skills, where the participants (speakers of the target language) analyzed the language errors in their interlocutors' language products (language learners) and further explained specific grammar structures, so as to develop their own grammar instruction skills [38]. In a study with a focus on teaching reflection, pre-service teachers exchanged information related to teacher training with others, which promoted their rethinking of past knowledge, and where they gained experience and practice with the process of obtaining/giving comments, so as to stimulate their reflections [34]. Finally, there were some studies in which participants, apart from cross-cultural learning, learned about different subjects (e.g., educational technology) [36]. Pre-service teachers with different cultural backgrounds exchanged information about major topics, such as technologies used in the class or how the teachers integrated these technologies into their curricula. These approaches helped them understand each other's degree of national technical support related to the educational process and also helped them learn how to integrate technologies into curricula derived from different cultural backgrounds [36].

Based on our results, we suggest that curricula should not focus only on cross-cultural learning. When learners are involved in cross-cultural projects, they can also learn other skills, such as linguistic skills, instructional skills, etc. For example, cross-cultural projects with speakers of the target language can help create authentic language environments. In addition, participants will be able to obtain knowledge, experience, and expertise related to pre-service teacher training based on diverse contexts and learners. Collaborative learning activities among pre-service teachers from different cultures can help them acquire cross-cultural collaborative skills.

The results showed that relatively few studies focused on cross-cultural awareness and sensitivity; however, many studies focused on cross-cultural understanding and competence. Shadiev et al. [30] argued that cross-cultural awareness and sensitivity are as important for cross-cultural learning as cross-cultural knowledge and competence. Therefore, we suggest that scholars pay attention to these two less explored competencies when designing their future studies. In addition, we suggest that other aspects of the crosscultural domain should be considered in future studies, e.g., cross-cultural adaptability, especially in the case of learners studying abroad.

In the field of linguistic skills, the reviewed studies mainly focused on the development of learners' communicative skills and linguistic awareness with the support of technology. Thus, we suggest that researchers explore how other abilities of language learners can be facilitated in technology-supported cross-cultural learning environments, e.g., vocabulary development. This is a very important ability because language learners need to have 
well-developed vocabularies to succeed in other skills such as reading, writing, listening, and speaking. In addition, successful language use should be diversified in terms of the target language. Most studies focused on English as a foreign or second language; therefore, other popular languages such as Chinese and French could be considered in future studies. Technology can help find and connect language learners from different parts of the world who have an interest in learning these popular languages.

Furthermore, domain knowledge can be also extended; for example, scholars may consider connecting cross-cultural learning with such subjects as history or geography since they are closely related to culture. For example, the Silk Road topic is worthy of consideration for learners who live along this famous road and learn about it in their history or geography classes. Learners from one part of this road (e.g., India) can learn about another part of the road (e.g., China) from learners residing there.

We found that in four studies, the participants in a cross-cultural learning project had no partners representing a foreign culture, so they had no opportunities for interaction or communication with people from the target culture [2]. In these four studies, all of the participants were from the same culture, and they learned about the target culture and acquired cultural knowledge from cultural information available in virtual environments, augmented reality environments [37], or via a telepresence robot [42]. Such advanced technologies created an immersive learning environment for cross-cultural learning that enhanced the learners' sense of presence. Even in the absence of interlocutors from the target culture, the learners were able to benefit to varying degrees from cultural immersion in virtual environments. Shih [2] pointed out that research focused on cross-cultural learning in environments supported by advanced technologies such as virtual, augmented, or mixed reality is still in its infancy. Therefore, we suggest that future researchers may wish to explore the benefits of such advanced technologies for cross-cultural learning and their effectiveness from a pedagogical point of view.

\subsection{Technologies}

The research question was "What technologies were applied in the cross-cultural studies?" Table 4 lists all technologies used for cross-cultural Learning in the reviewed studies. Our results show that technologies can be used for (1) communication among participants, (2) creating communication content or cultural artifacts, and (3) supporting communication. For example, learners from different cultures in Hsu and Beasley [28] used Skype to communicate with each other and exchange culture-related information. Learners in Lee [32] created media products about their culture using Movie Maker and shared their projects with their partners. As for supporting communication, learners used SELT to translate their communication content in their mother tongue into the languages of their partners because they had no common language by which to communicate [1], or learners uploaded their files on cloud for sharing purposes using Dropbox because email or instant messaging applications do not allow sending large files [39].

Furthermore, as can be seen from the table, these technological tools can be used to support synchronous (e.g., Skype) or asynchronous (e.g., e-mail) communication. Most of the reviewed studies used more than one tool to support the cross-cultural learning process. An earlier review study [9] found that almost all researchers used asynchronous communication tools, and with the development of technology, in subsequent published studies, researchers began to use a combination of synchronous and asynchronous communication. It is worth mentioning that in most studies using a combination of synchronous and asynchronous communication, the researchers tended to help participants understand each other through asynchronous communication in the first steps of the learning activity. In this case, the advantage of asynchronous communication could be fully utilized, thus making communication efficient. For example, asynchronous communication is useful in the beginning of the cross-cultural learning process because the learners are not yet acquainted with one another; therefore, it helps to decrease their anxiety and reduce their inhibitions [29]. In addition, asynchronous communication improves motivation to dis- 
close personal information, which is problematic for some learners during synchronous communication [21]. Furthermore, communicating asynchronously allows the learners to have more time to post their information, read the posts of others, ask or answer questions, and also to communicate at convenient times [39]. On the other hand, synchronous communication is useful because people can instantly communicate and exchange culture-related information, reflect on experiences, and offer or receive comments.

In addition, we found that related studies published after 2014 reported on new technologies that were not mentioned in related studies published before 2014. On the other hand, some technologies mentioned in studies before 2014 were no longer mentioned in studies published after 2014. For example, speech-enabled language translation technology has existed for a long time, but because speech recognition and computer-aided translation has improved greatly in the last few years, scholars have not used it until now. This technology is important because it can be used to bridge the language gap among participants from different cultures. Since this type of technology was not observed in the past reviewed studies, we suggest considering it for further practice and research because the use of this technology can provide an interesting direction of research in the field of interculturality. With the rapid development of technology, more powerful and immersive technologies have also emerged, such as wearable technology, virtual, augmented or mixed reality, and so on. These technologies can be used to make the cross-cultural learning environment more immersive and authentic as well as to provide a better sense of presence.

Based on our results, we suggest that educators and researchers use various technological tools for supporting communication and content creation during cross-cultural learning. Both synchronous and asynchronous communication can be implemented for interaction and information exchanges among participants because each of these types of communication has its own advantages. Furthermore, the use of emerging learning technologies is suggested for supporting cross-cultural learning. For example, Bonk and Wiley [48] listed an assortment of popular tools like Facebook Messenger Rooms, Google Meet, Microsoft Teams and Zoom for connections among learners that received interest from educators and researchers nowadays, when we are dealing with a global pandemic. Other emerging learning technologies such as virtual or augmented reality enable participants from different countries to communicate with each other and help them truly appreciate the culture of other countries in a more immersive environment.

\subsection{Methodology and Findings of the Reviewed Studies}

In this subsection, we discuss the results to our fourth research questions "What methodology was used in the cross-cultural studies, and what were their main findings?" Our results showed that in seven of the reviewed studies, the age of the participants was not shown; in others, participants' age ranged from 10 to 44 years old. In terms of participants' academic status, our results showed that most studies were conducted with university and high school students [35]. Based on our results, we suggest that researchers indicate the participants' demographics, such as age and academic level. Such information can be useful to interpret the effect of technology application and add validity to research conclusions [13].

Our results showed that most studies adopted qualitative and quantitative methodologies or only qualitative or quantitative methodology. This finding suggests that many studies used qualitative and quantitative methods to make their conclusions more powerful. Our review results indicated that some studies used survey data from open-ended questions to conduct their quantitative analyses. Learners were surveyed about their experiences related to learning and usage of technology in order to assess learner development or to determine the usefulness of a given technology. In addition, it is worth noting that the data collected in five studies included reflective data from learners. For example, learners were asked to describe and reflect on their learning experiences, and then scholars analyzed and coded the content of the reflective data based on Byram's assessment criteria to understand the degree of cross-cultural development in the learners [28]. However, 
qualitative data collected from learners are subject to bias. Furthermore, measuring different dimensions of cross-cultural learning (e.g., knowledge, sensitivity, and awareness) cannot be limited to a single instrument due to the complexity of research constructs. Therefore, in future studies, we suggest collecting both qualitative and quantitative data. This approach will allow triangulating data from different data sources and will make the evidence more comprehensive and convincing. For example, researchers may determine the applicability of a technology to cross-cultural learning through a controlled experiment first, e.g., involving a control group to compare cross-cultural learning achievements with an intervention group. Then, participants could be surveyed regarding their perceptions and opinions towards the technological intervention, and the results could then be used to support the findings obtained through the quantitative approach, or vice versa. It is also suggested that researchers apply the reflective learning method to assist with cross-cultural learning. Future researchers may also wish to use some other data. For example, it is possible to collect physiological data such as anxiety or emotions using wearables such as NeuroSky MindWave [49] or smartwatches [50]. These tools can help measure how learning activities supported by a given technology may assist in decreasing anxiety and evoking positive emotions in learners, thus further stimulating learning motivation.

Our results showed that most studies had positive results regarding the support of technology in the cross-cultural domain as well as in other domains, such as FL/SL learning or instructional skills. For example, Jin [26] reported Facebook to be useful in terms of facilitating intercultural interactions and competence. Chen and Yang [25] found that intercultural language learning enabled the learners in their study to experience authentic language learning that fostered linguistic competence. The results obtained by Angelova and Zhao [38] showed that an online collaborative project led to improvements in the teaching skills of speakers of English as a result of tutoring learners of English.

Based on the results, we suggest that researchers take the results of the reviewed studies into consideration when designing future studies. That is, factors affecting crosscultural learning, the role of peer feedback, the usefulness of learning strategies, error correction approaches, cross-cultural online collaborative learning, reflective feedback, as well as possible issues and their solutions can inform the design of future studies. For example, when factors related to the critical success of online international learning exchanges are determined (e.g., self-efficacy, the exchange infrastructure, and the quality of the exchange activities), educators and researchers can establish effective implementation strategies for cross-cultural learning. In addition, critical success factors can be effectively managed and controlled as part of a successful implementation strategy for cross-cultural learning projects in order to obtain positive outcomes, such as learning satisfaction, foreign language capability, and intercultural competence [41].

\section{Conclusions}

In this study, we reviewed articles on technology-supported cross-cultural learning. Our results showed that the reviewed studies built their research frameworks mostly based on Byram's model and the cultural convergence theory. The reviewed studies could be categorized in terms of the curricula focus into (a) cross-cultural learning, (b) linguistic skills, and (c) pre-service teacher training. We also found that the most frequently used technologies to support cross-cultural learning were Skype, e-mail, and blogs. Compared to earlier review studies, we identified several new technologies never mentioned before 2014, as well as old technologies never mentioned after 2014 that were intended to support cross-cultural learning. We discovered that the data in most of the reviewed studies comprised a combination of both qualitative and quantitative data. Most of the reviewed studies reported the role of technology in cross-cultural learning and how technologies can support FL/SL learning and pre-service teacher training. Finally, the reviewed studies pointed out several issues encountered during the cross-cultural learning process and suggested corresponding solutions. Based on our results, we derived several implications and offered suggestions for teaching and the research community in the field. There- 
fore, the results of our review study can help educators and researchers understand the current research status of technology-supported cross-cultural learning, and to design and implement related learning projects more efficiently. For example, in terms of theoretical foundation, knowing, understanding, and using it appropriately is important in any empirical studies. Knowledge about curricula from reviewed studies can serve as a guiding framework for designing and preparing instructional content of future research. Our results related to technologies can be helpful to educators and researchers in selecting appropriate tools based on participants' academic level, interests and curriculum goals. Finally, methodology may serve as a blueprint for further studies and findings obtained from earlier research may inspire educators and researchers about what to expect from cross-cultural learning projects.

This review study has one limitation that should be acknowledged. Twenty-three articles reviewed in this study were among those published in the top nineteen SSCI journals. That is, conference papers, book reviews, dissertations, etc., were excluded from this review. For this reason, our results cannot be broadly generalized because we reviewed a small number of articles. Future studies may consider this limitation and extend their reviews by including more related articles published in a greater variety of references. This can be done by expanding the selection criteria, e.g., including articles with a high number of citations. Other future research directions are as follows: using other theoretical foundations for cross-cultural studies (e.g., the contact hypothesis, the community of inquiry model, etc.), designing other types of culture-related learning (e.g., between cultures that are situated within one country), and focusing on competencies (e.g., cross-cultural awareness and sensitivity) that have received a little attention in the field. Scholars may also use advanced technologies such as virtual, augmented, or mixed reality for the purpose of creating more immersive and authentic learning environments. Furthermore, scholars may explore the affective domain (e.g., anxiety) by collecting physiological data.

Author Contributions: Methodology, conceptualization, investigation, formal analysis, X.W. and R.S.; validation, T.-T.W.; original draft preparation, X.W.; review and editing, R.S., T.-T.W. and Y.-M.H.; supervision, R.S. and Y.-M.H. All authors have read and agreed to the published version of the manuscript.

Funding: This research received no external funding.

Institutional Review Board Statement: Not applicable.

Informed Consent Statement: Not applicable.

Data Availability Statement: The data presented in this study are available on request from the corresponding author. The data are not publicly available due to ethical reasons.

Acknowledgments: Not available.

Conflicts of Interest: The authors declare no conflict of interest.

\section{Appendix A. List of Top Nineteen SSCI Journals Related to Technology}

1. Internet and Higher Education;

2. Computers \& Education;

3. International Journal of Computer-Supported Collaborative Learning;

4. Learning Media and Technology;

5. British Journal of Educational Technology;

6. ReCALL;

7. Language Learning \& Technology;

8. Computer Assisted Language Learning;

9. IEEE Transactions on Learning Technologies;

10. Journal of Computer Assisted Learning;

11. International Review of Research in Open and Distributed Learning;

12. Education Technology \& Society; 


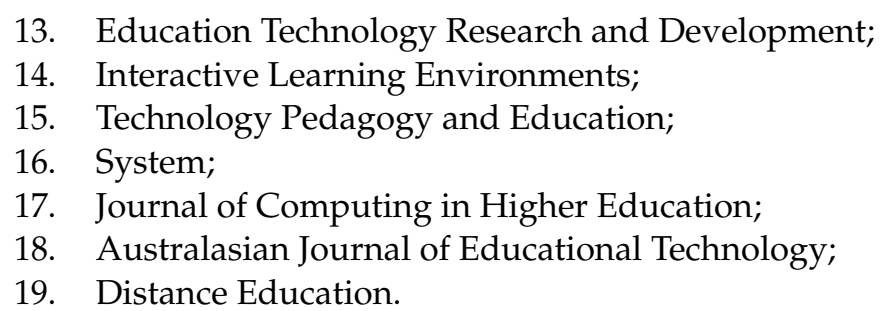

\section{References}

1. Shadiev, R.; Huang, Y.M. Facilitating cross-cultural understanding with learning activities supported by speech-to-text recognition and computer-aided translation. Comput. Educ. 2016, 98, 130-141. [CrossRef]

2. Shih, Y.-C. A virtual walk through London: Culture learning through a cultural immersion experience. Comput. Assist. Lang. Learn. 2013, 28, 1-22. [CrossRef]

3. Shadiev, R.; Wu, T.-T.; Sun, A.; Huang, Y.-M. Applications of speech-to-text recognition and computer-aided translation for facilitating cross-cultural learning through a learning activity: Issues and their solutions. Educ. Technol. Res. Dev. 2017, 66, 191-214. [CrossRef]

4. Biasutti, M. The student experience of a collaborative e-learning university module. Comput. Educ. 2011, 57, 1865-1875. [CrossRef]

5. Walther, J.B.; Hoter, E.; Ganayem, A.; Shonfeld, M. Computer-mediated communication and the reduction of prejudice: A controlled longitudinal field experiment among Jews and Arabs in Israel. Comput. Hum. Behav. 2015, 52, 550-558. [CrossRef]

6. Wang, C. Instructional design for cross-cultural online collaboration: Grouping strategies and assignment design. Australas. J. Educ. Technol. 2011, 27, 243-258. [CrossRef]

7. Biasutti, M. A coding scheme to analyse the online asynchronous discussion forums of university students. Technol. Pedagog. Educ. 2017, 26, 601-615. [CrossRef]

8. Biasutti, M. A comparative analysis of forums and wikis as tools for online collaborative learning. Comput. Educ. 2017, 111, 158-171. [CrossRef]

9. Çiftçi, E.Y. A review of research on intercultural learning through computer-based digital technologies. Educ. Technol. Soc. 2016, 19, 313-327.

10. Lee, L.; Markey, A. A study of learners' perceptions of online intercultural exchange through Web 2.0 technologies. Recall 2014, 26, 281-297. [CrossRef]

11. Chun, D. Language and culture learning in higher education via telecollaboration. Pedagog. Int. J. 2015, 10, 5-21. [CrossRef]

12. Golonka, E.M.; Bowles, A.R.; Frank, V.M.; Richardson, D.L.; Freynik, S. Technologies for foreign language learning: A review of technology types and their effectiveness. Comput. Assist. Lang. Learn. 2012, 27, 70-105. [CrossRef]

13. O'Dowd, R. Emerging Trends and New Directions in Telecollaborative Learning. CALICO J. 2015, 33, 291-310. [CrossRef]

14. Çiftçi, E.Y.; Savaş, P. The role of telecollaboration in language and intercultural learning: A synthesis of studies published between 2010 and 2015. Recall 2018, 30, 278-298. [CrossRef]

15. Avgousti, M.I. Intercultural communicative competence and online exchanges: A systematic review. Comput. Assist. Lang. Learn. 2018, 31, 819-853. [CrossRef]

16. Lewis, T.; O’Dowd, R. Online intercultural exchange and foreign language learning: A systematic review. In Online Intercultural Exchange: Policy, Pedagogy, Practice; O’Dowd, R., Lewis, T., Eds.; Routledge: New York, NY, USA, 2016; pp. 21-68.

17. Piri, S.; Riahi, S. Cross-cultural perspectives on technology-enhanced language learning: A review of research. In Cross-Cultural Perspectives on Technology-Enhanced Language Learning; Tafazoli, D., Parra, M.E.G., Huertas-Abril, C.A., Eds.; IGI Global: Hershey, PA, USA, 2018; pp. 1-15.

18. Moher, D.; Shamseer, L.; Clarke, M.; Ghersi, D.; Liberati, A.; Petticrew, M.; Shekelle, P.; Stewart, L.A.; PRISMA-P Group. Preferred reporting items for systematic review and meta-analysis protocols (PRISMA-P) 2015 statement. Syst. Rev. 2015, 4, 1-9. [CrossRef]

19. Crompton, H.; Burke, D.; Lin, Y.-C. Mobile learning and student cognition: A systematic review of PK-12 research using Bloom's Taxonomy. Br. J. Educ. Technol. 2019, 50, 684-701. [CrossRef]

20. Kukulska-Hulme, A.; Viberg, O. Mobile collaborative language learning: State of the art. Br. J. Educ. Technol. 2018, 49, 207-218. [CrossRef]

21. Shadiev, R.; Liu, T.; Hwang, W.-Y. Review of research on mobile-assisted language learning in familiar, authentic environments. Br. J. Educ. Technol. 2019, 51, 709-720. [CrossRef]

22. Viberg, O.; Hatakka, M.; Bälter, O.; Mavroudi, A. The current landscape of learning analytics in higher education. Comput. Hum. Behav. 2018, 89, 98-110. [CrossRef]

23. Viberg, O.; Andersson, A.; Wiklund, M. Designing for sustainable mobile learning-Re-evaluating the concepts "formal" and "informal". Interact. Learn. Environ. 2018, 1-12. [CrossRef]

24. Creswell, J.W. Educational Research: Planning, Conducting, and Evaluating Quantitative and Qualitative Research; Pearson Education: Boston, MA, USA, 2014.

25. Chen, J.J.; Yang, S.C. Fostering foreign language learning through technology-enhanced intercultural projects. Lang. Learn. Technol. 2014, 18, 57-75. [CrossRef] 
26. Jin, S. Using Facebook to promote Korean EFL learners' intercultural competence. Lang. Learn. Technol. 2015, 19, 38-51. [CrossRef]

27. Chen, Y.J.; Yang, S.C. Promoting cross-cultural understanding and language use in research-oriented Internet-mediated intercultural exchange. Comput. Assist. Lang. Learn. 2016, 29, 262-288. [CrossRef]

28. Hsu, S.Y.S.; Beasley, R.E. The effects of international email and Skype interactions on computer-mediated communication perceptions and attitudes and intercultural competence in Taiwanese students. Australas. J. Educ. Technol. 2019, 35, 149-162. [CrossRef]

29. Shadiev, R.; Hwang, W.Y.; Huang, Y.M. A pilot study: Facilitating cross-cultural understanding with project-based collaborative learning in an online environment. Australas. J. Educ. Technol. 2015, 31, 123-139. [CrossRef]

30. Shadiev, R.; Sun, A.; Huang, Y. A study of the facilitation of cross-cultural understanding and intercultural sensitivity using speech-enabled language translation technology. Br. J. Educ. Technol. 2018, 50, 1415-1433. [CrossRef]

31. Yang, R. The use of questions in a synchronous intercultural online exchange project. Recall 2017, 30, 112-130. [CrossRef]

32. Lee, K. Implementing computer-mediated intercultural communication in English education: A critical reflection on its pedagogical challenges. J. Comput. Assist. Learn. 2018, 34, 673-687. [CrossRef]

33. Wu, Z. Positioning (mis)aligned: The (un)making of intercultural asynchronous computer-mediated communication. Lang. Learn. Technol. 2018, 22, 75-94. [CrossRef]

34. Wach, A. Promoting pre-service teachers' reflections through a cross-cultural keypal project. Lang. Learn. Technol. 2015, 19, 34-45. [CrossRef]

35. Schenker, T. Syntactic complexity in a cross-cultural E-mail exchange. System 2016, 63, 40-50. [CrossRef]

36. Yang, J.; Kinshuk, Y.H.; Chen, S.J.; Huang, R. Strategies for Smooth and Effective Cross-Cultural Online Collaborative Learning. Educ. Technol. Soc. 2014, 17, 208-221. [CrossRef]

37. Yang, M.T.; Liao, W.C. Computer-Assisted Culture Learning in an Online Augmented Reality Environment Based on Free-Hand Gesture Interaction. IEEE Trans. Learn. Technol. 2014, 7, 107-117. [CrossRef]

38. Angelova, M.; Zhao, Y. Using an online collaborative project between American and Chinese students to develop ESL teaching skills, cross-cultural awareness and language skills. Comput. Assist. Lang. Learn. 2014, 29, 167-185. [CrossRef]

39. Bueno-Alastuey, M.C.; Kleban, M. Matching linguistic and pedagogical objectives in a telecollaboration project: A case study. Comput. Assist. Lang. Learn. 2016, 29, 148-166. [CrossRef]

40. Coffey, A.J.; Kamhawi, R.; Fishwick, P.; Henderson, J. The efficacy of an immersive 3D virtual versus 2D web environment in intercultural sensitivity acquisition. Educ. Technol. Res. Dev. 2017, 65, 455-479. [CrossRef]

41. Lee, J.; Park, S. Analysis of critical success factors of online international learning exchange of Korean school pupils with English-speaking counterparts. Br. J. Educ. Technol. 2016, 48, 1228-1238. [CrossRef]

42. Liao, J.; Lu, X. Exploring the affordances of telepresence robots in foreign language learning. Lang. Learn. Technol. 2018, 22, 20-32. [CrossRef]

43. Melo-Pfeifer, S. Blogs and the development of plurilingual and intercultural competence: Report of a co-actional approach in Portuguese foreign language classroom. Comput. Assist. Lang. Learn. 2013, 28, 220-240. [CrossRef]

44. Byram, M. Teaching and Assessing Intercultural Communicative Competence; Multilingual Matters: Clevedon, UK, 1997.

45. Garrison, D.R.; Arbaugh, J. Researching the community of inquiry framework: Review, issues, and future directions. Internet High. Educ. 2007, 10, 157-172. [CrossRef]

46. Kilis, S.; Yıldırım, Z. Investigation of community of inquiry framework in regard to self-regulation, metacognition and motivation. Comput. Educ. 2018, 126, 53-64. [CrossRef]

47. Allport, G.W. The Nature of Prejudice; Addison Wesley: Reading, MA, USA, 1954.

48. Bonk, C.J.; Wiley, D.A. Preface: Reflections on the waves of emerging learning technologies. Educ. Technol. Res. Dev. 2020, 68, 1595-1612. [CrossRef] [PubMed]

49. Hsu, L. To CALL or not to CALL: Empirical evidence from neuroscience. Comput. Assist. Lang. Learn. 2020, 1-24. [CrossRef]

50. Shadiev, R.; Hwang, W.Y.; Liu, T.Y. A study of the use of wearable devices for Healthy and enjoyable English as a Foreign language learning in authentic contexts. Educ. Technol. Soc. 2018, 21, 217-231. 